\title{
Multi-Field-Coupled Model and Solution of Active Electronically Scanned Array Antenna Based on Model Reconstruction
}

\author{
Dan Han $\mathbb{D}^{1},{ }^{1}$ Jin Huang $\left(\mathbb{D},{ }^{1}\right.$ Jinzhu Zhou $\mathbb{D}^{1},{ }^{1}$ Mei Wang, ${ }^{2}$ Sen Xu, ${ }^{1}$ Haitao Li $\left(\mathbb{D},{ }^{1}\right.$ and Shen $\mathrm{Li}^{1}$ \\ ${ }^{1}$ Key Laboratory of Electronic Equipment Structure Design, Ministry of Education, Xidian University, Xi'an 710071, China \\ ${ }^{2}$ Structure Department, No. 38 Research Institute of CETC, Hefei 230031, China \\ Correspondence should be addressed to Jin Huang; jhuang@mail.xidian.edu.cn
}

Received 19 June 2018; Accepted 30 July 2018; Published 27 November 2018

Academic Editor: Francesco D'Agostino

Copyright @ 2018 Dan Han et al. This is an open access article distributed under the Creative Commons Attribution License, which permits unrestricted use, distribution, and reproduction in any medium, provided the original work is properly cited.

\begin{abstract}
Active electronically scanned array antenna (AESA antenna) is capable of controlling the radiation pattern by controlling the feeding phase of the radiating elements. It has good performance and plays an important role in radar systems. With the development of AESA antenna towards high-frequency bands and high-density arrays, the structural-electromagnetic-thermal (SET) coupling becomes increasingly significant. It seriously restricts the realization of high performances of AESA antennas. However, the previously reported theoretical multi-field-coupled model for the coupling problem ignores the effect of the deformations of the feed system and array elements on the electrical performance. It only considers the positional deviations of the array elements in the coupling field. As a result, the accuracy of the numerical solution by the theoretical model is reduced. To overcome the above problems, this paper first establishes the field-circuit coupling model by introducing the deformation errors of the feed system into the existing theoretical model. Secondly, this paper proposes a new numerical solution for the multi-field-coupled problem of AESA antennas based on model reconstruction. And the model reconstruction includes the following: the NURBS (nonuniform rational B-spline) surface fitting algorithm that completes the mapping from finite element models to geometric models by the surface equations established by the node information and the local model reconstruction algorithm that determines the local geometric models by the positions and the directions. The NURBS surface fitting algorithm guarantees the accuracy of both the positions and shapes of array elements. The local model reconstruction algorithm ensures the accuracy of the amplitudes and phases of feed connectors. Finally, the numerical solution was applied to the 32-element AESA antenna and the simulations are close to the measurements.
\end{abstract}

\section{Introduction}

Active electronically scanned array antenna (AESA antenna) has high reliability, multiple functions, strong detection capability, and good stealth capability $[1,2]$. In the past several decades, AESA antennas played an important role in civilian operations and the military arena [3]. To obtain better performance, the AESA antenna is developing towards higher frequency and higher density. The technological developments of meter wave and centimeter wave detection radars are relatively mature. Millimeter waves are widely used in communication systems. The frequency of highspeed and short-distance transmission equipment can reach up to dozens of gigahertz $(\mathrm{GHz})$. The research of higher terahertz application has already begun. The frequency band of radio waves observed in radio astronomy can reach up to hundreds of gigahertz $(\mathrm{GHz})$ [4]. Moreover, the assembly density of AESA antennas continues to increase with the transition from two-dimensional assembly to three-dimensional assembly. Accordingly, the volume of equipment must become increasingly small. For example, the size of radio frequency systems has been reduced from $0.03 \mathrm{~m}^{3}$ to $0.001 \mathrm{~m}^{3}$ [4]. Thus, the structural-electromagnetic-thermal (SET) coupling becomes more and more significant. It causes the gain loss, sidelobe level upgrade, and inaccurate beam pointing $[5,6]$, thus restricting the development of high-performance array antennas. Therefore, the multifield coupling is a crucial problem that needs to be solved [7].

There are two major types of numerical solutions previously reported for the multifield coupling of AESA antennas. 


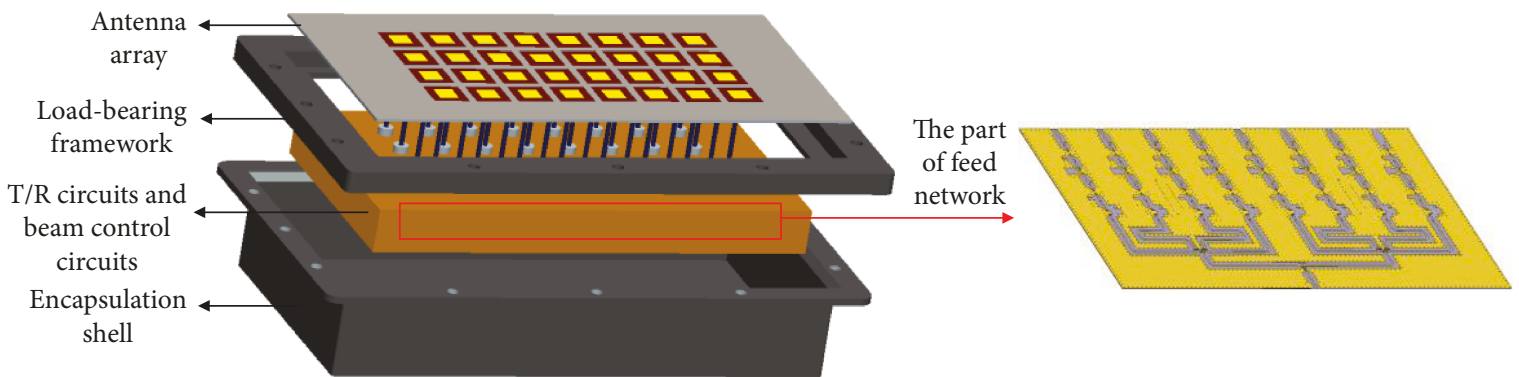

Figure 1: Structural configuration of the AESA antenna.

One is by the theoretical multi-field-coupled model [8, 9]. However, the model ignores the effect of the deformations of the feed system and array elements on the electrical performance. It only considers the positional deviations of the array elements in the coupling field [9], thus reducing the accuracy of the numerical solution. The other is by simulations. However, between different software in coupling fields, the data models are heterogeneous and the mesh of the finite element model does not match because of different analysis purposes and tools. To overcome the above problems in simulation, two approaches came into being. One approach is importing the heterogeneous mesh into the simulation software directly $[10,11]$. However, the imported mesh must be simplified, refined, and homogenized to meet the analysis requirements and the process is complicated [12]. The other approach is fitting a new solid model from the heterogeneous mesh and then remeshing on the new model [13-15]. However, new errors are introduced in the fitting process and the accuracy of the result of the electromagnetic analysis is reduced [12].

For the above problems, this paper first establishes the field-circuit coupling model by introducing the deformation errors of the feed system into the existing theoretical model. Secondly, this paper proposes a new simulation method based on model reconstruction that precisely reflects both the positional deviations and self-deformations of the array elements. One of the key benefits of the method is passing the solid model between different simulation tools in coupling fields and remesh on the new model, which avoids the tedious meshing process. Moreover, the NURBS (nonuniform rational B-spline) surface fitting algorithm guarantees the accuracy of both the positions and shapes of array elements. And the local model reconstruction algorithm ensures the accuracy of the amplitudes and phases of feed connectors. Thus, the accuracy of the electromagnetic analysis is improved. Figure 1 shows the structure of the AESA antenna. And the antenna array and feed network that this paper concerns about are shown in the figure.

\section{Multi-Field-Coupled Model and Solution of AESA Antennas}

The multifield coupling of the AESA antenna is primarily the sequential coupling among the temperature field, the structural displacement field, and the electromagnetic field. As shown in Figure 2, structural deformations are caused by environmental loads and changes of structural parameters, also known as structural displacement field (path (1)). Secondly, the structural displacement field affects the temperature field and the electromagnetic field through the above deformations (path (2)). Finally, the influence of the temperature field has two forms: one is the impact on the performance of electromagnetic devices (such as T/R components) (path (3) and the other is the impact on the electromagnetic field by the changes of the structural displacement field (path (4)) [16].

2.1. Field-Circuit Coupling Model. For the above threefield coupling process of AESA antennas, in the traditional three-field coupling model $[8,9]$, as shown in (1), the excitation source $I_{m n}^{\prime}$ by the feed system was ideally processed, often by applying a unit excitation source or an excitation source that satisfies the Taylor distribution. However, in the coupling field, the feed system also deforms. Moreover, the electrical performance is affected by the changed characteristic impedance and power ratio of the feed network, thereby affecting the excitation current. In addition, in the feed network, the $\lambda / 4$ impedance converter has a narrow line width because of the high transmission impedance. And in order to reduce the coupling effect, the spacing of its lines is large. Therefore, in the coupling field, the sensitivity of deformation error of the $\lambda / 4$ impedance converter is large. In this paper, the relation between the length variation $\Delta l$ of the $\lambda / 4$ impedance converter in the feed network and the excitation current $I_{m n}^{\prime}$ is deduced. And this relation is introduced into the existing coupling model to establish the fieldcircuit coupling model. Figure 3 shows the spatial relation of the AESA antenna.

$$
\begin{aligned}
E(\theta, \phi)= & \sum_{m=0}^{M-1} \sum_{n=0}^{N-1} E_{m n}^{\prime}(\theta, \phi) I_{m n}^{\prime} \\
& \times \exp \left\{j k \left[\left(m d_{x}+\sum_{i=0}^{m} \Delta x_{i j}(\delta, T)\right) f_{x}(\theta, \phi)\right.\right. \\
& +\left(n d_{y}+\sum_{j=0}^{n} \Delta y_{i j}(\delta, T)\right) f_{y}(\theta, \phi) \\
& \left.\left.+\Delta z_{i j}(\delta, T) f_{z}(\theta)\right]+j S_{m n}(T)\right\}
\end{aligned}
$$



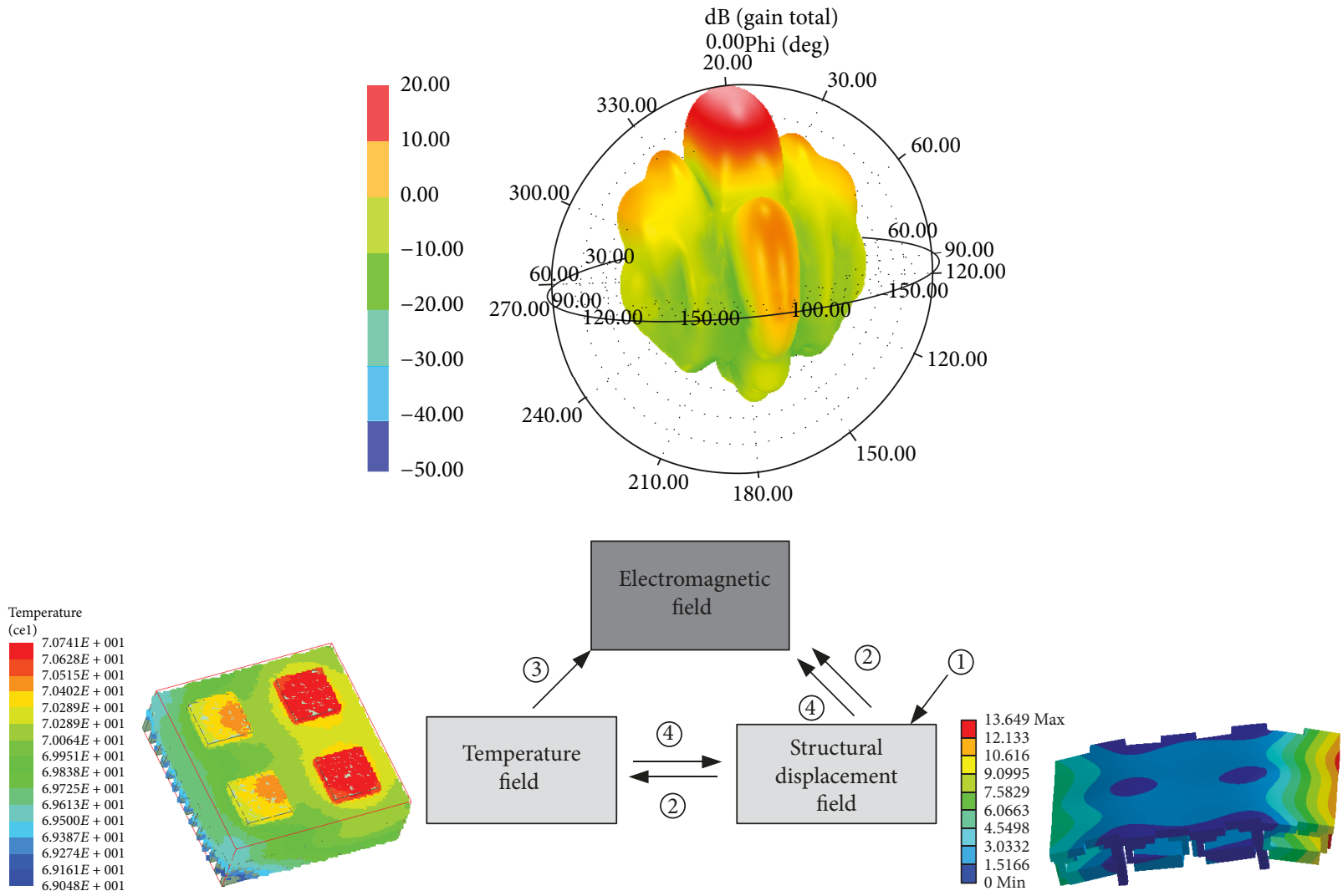

FIgURE 2: Three-field coupling process of the AESA antenna.
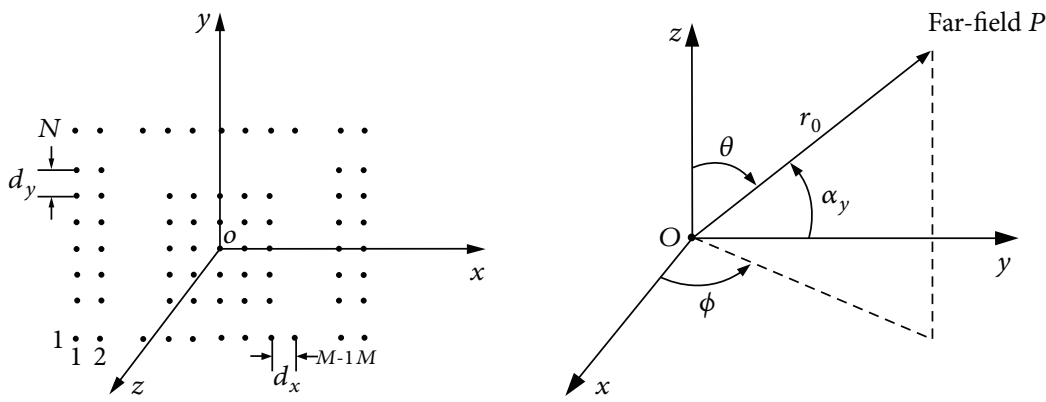

FIgURE 3: Spatial relation of the AESA antenna.

where $E(\theta, \phi)$ is the pattern function, $E_{m n}^{\prime}(\theta, \phi)$ is the pattern function of the radiating elements in an antenna array, $I_{m n}^{\prime}$ is the amplitude of excitation current, $T$ is the structure temperature, $\delta\left(\beta_{1}, \beta_{2}, \ldots, \beta_{R}\right)$ is the structural displacement, $\beta_{i}$ $(i=1,2, \ldots, R)$ are the structural design variables, $f_{x}(\theta, \phi)$, $f_{y}(\theta, \phi)$, and $f_{z}(\theta)$ are functions of element position and direction determined by the array arrangement form, such as the hexagonal AESA antenna and rectangular AESA antenna, $\Delta x_{i j}(\delta, T), \Delta y_{i j}(\delta, T)$, and $\Delta z_{i j}(\delta, T)$ are the deviation values of the element position determined by $T$ and $\delta$, $S_{m n}(T)$ is the array phase difference controlled by the phase shifter affected by $T$, and $d_{x}$ and $d_{y}$ are the intervals of the radiating elements.
Figure 4(a) shows the circuit parameters of the power divider in the feed network. Next, the relation between $\Delta l$ and $I_{m n}^{\prime}$ is deduced from two aspects. First, the deformation of the microstrip causes impedance mismatching of the power divider, which results in the nonzero reflection coefficient at the power input end $P_{\text {in }}$.

$$
P_{\text {in }}=P_{0}\left(1-\left|\Gamma_{\text {in }}\right|^{2}\right)
$$

Secondly, the deformation of the microstrip causes the uneven distribution of the power on each branch, assuming that the input power on one of the branches $P_{i n B}=K P_{\text {in }}$. 


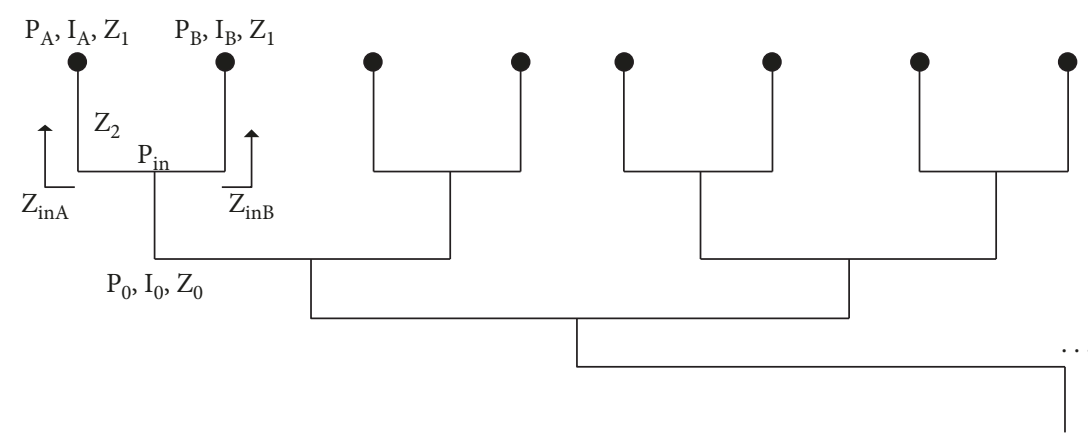

(a) Diagram of circuit parameters

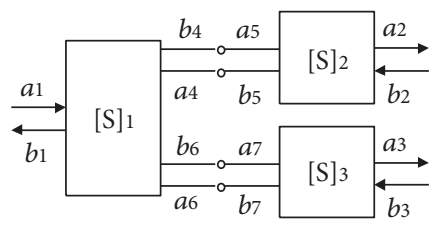

(b) Three-port network

Figure 4: Diagram of the power divider.

And the $K$ was deduced according to the impedance converter theory as

$$
K=\frac{Z_{i n A}}{Z_{i n A}+Z_{i n B}},
$$

and thus,

$$
P_{\text {inA }}=(1-K) P_{\text {in }} .
$$

At the same time, considering that there is also the reflection between the $\lambda / 4$ impedance converter and the load, thus, the load power

$$
\begin{aligned}
& P_{A}=P_{i n A}\left(1-\left|\Gamma_{i n A}\right|^{2}\right), \\
& P_{B}=P_{i n B}\left(1-\left|\Gamma_{i n B}\right|^{2}\right) .
\end{aligned}
$$

Finally, according to the relation between the current and the power, the element excitation current is obtained as

$$
\begin{aligned}
I_{m n}^{\prime}= & {\left[P_{0} \times\left(1-\left|\frac{Z_{m n}\left(\Delta l_{m n}\right) Z_{m n^{\prime}}\left(\Delta l_{m n}\right)-Z_{0}\left(Z_{m n}\left(\Delta l_{m n}\right)+Z_{m n^{\prime}}\left(\Delta l_{m n}\right)\right)}{Z_{m n}\left(\Delta l_{m n}\right) Z_{m n^{\prime}}\left(\Delta l_{m n}\right)+Z_{0}\left(Z_{m n}\left(\Delta l_{m n}\right)+Z_{m n^{\prime}}\left(\Delta l_{m n}\right)\right)}\right|^{2}\right)\right.} \\
& \left.\times\left(1-\left|\frac{Z_{m n}^{\prime}\left(\Delta l_{m n}\right)-Z_{l}}{Z_{m n}{ }^{\prime}\left(\Delta l_{m n}\right)+Z_{l}}\right|^{2}\right) \times \frac{Z_{m n^{\prime}}\left(\Delta l_{m n}\right)}{Z_{m n}\left(\Delta l_{m n}\right)+Z_{m n^{\prime}}\left(\Delta l_{m n}\right)}\right]^{1 / 2} \times Z_{l}^{-1 / 2},
\end{aligned}
$$

where $P_{0}$ is the total power at the input of the power divider, $Z_{0}$ is the characteristic impedance of the microstrip at the input end, $Z_{l}$ is the impedance of the array element, $Z_{m n}$ and $Z_{m n^{\prime}}$ (also $Z_{i n A}$ or $Z_{i n B}$ in the figure) are the impedance of the $\lambda / 4$ impedance converter looking into the load from the input end (the arrow pointing), and $Z_{m n}{ }^{\prime}$ is the impedance of the $\lambda / 4$ impedance converter looking into the source from the output end (the opposite direction of the arrow). $Z_{m n}$ is shown as

$$
Z_{m n}=Z_{2} \frac{Z_{l}+j Z_{2} \tan \left(\beta\left(l+\Delta l_{m n}\right)\right)}{Z_{2}+j Z_{l} \tan \left(\beta\left(l+\Delta l_{m n}\right)\right)} .
$$

$Z_{m n^{\prime}}$ and $Z_{m n}{ }^{\prime}$ are the same, where $Z_{2}$ is the characteristic impedance of the impedance converter. And the meaning of $n^{\prime}$ in the formula is as follows:

$$
n^{\prime}= \begin{cases}n+1, & n \text { is an odd number } \\ n-1, & n \text { is an even number. }\end{cases}
$$

If the above power divider is equivalent to the three-port network, as shown in Figure 4(b), the cascaded microwave full matrix is

$$
\left[\begin{array}{c}
b_{1} \\
b_{2} \\
b_{3} \\
b_{4} \\
\vdots \\
b_{7}
\end{array}\right]=\left[\begin{array}{ccc:ccc}
s_{11} & s_{12} & s_{13} & s_{14} & \cdots & s_{17} \\
s_{21} & s_{22} & s_{23} & \cdots & \cdots & \cdots \\
s_{31} & s_{32} & s_{33} & s_{34} & \cdots & s_{37} \\
\hdashline s_{41} & \cdots & s_{43} & s_{44} & \cdots & s_{47} \\
\cdots & \cdots & \cdots & \cdots & \cdots & \cdots \\
s_{71} & \cdots & s_{73} & s_{74} & \cdots & s_{77}
\end{array}\right]\left[\begin{array}{c}
a_{1} \\
a_{2} \\
a_{3} \\
a_{4} \\
\vdots \\
a_{7}
\end{array}\right]
$$

Thus, the scattering parameter matrix of the three-port network is

$$
S=S_{\text {II }}+S_{\text {III }}\left(\beta-S_{\text {II II }}\right)^{-1} S_{\text {II I }} \text {, }
$$

where $S_{\text {II }}, S_{\text {III }}, S_{\text {III }}$, and $S_{\text {II II }}$ are the blocked submatrix.

2.2. Numerical Solution for Multifield Coupling Problem. The multifield coupling of the AESA antenna is primarily 


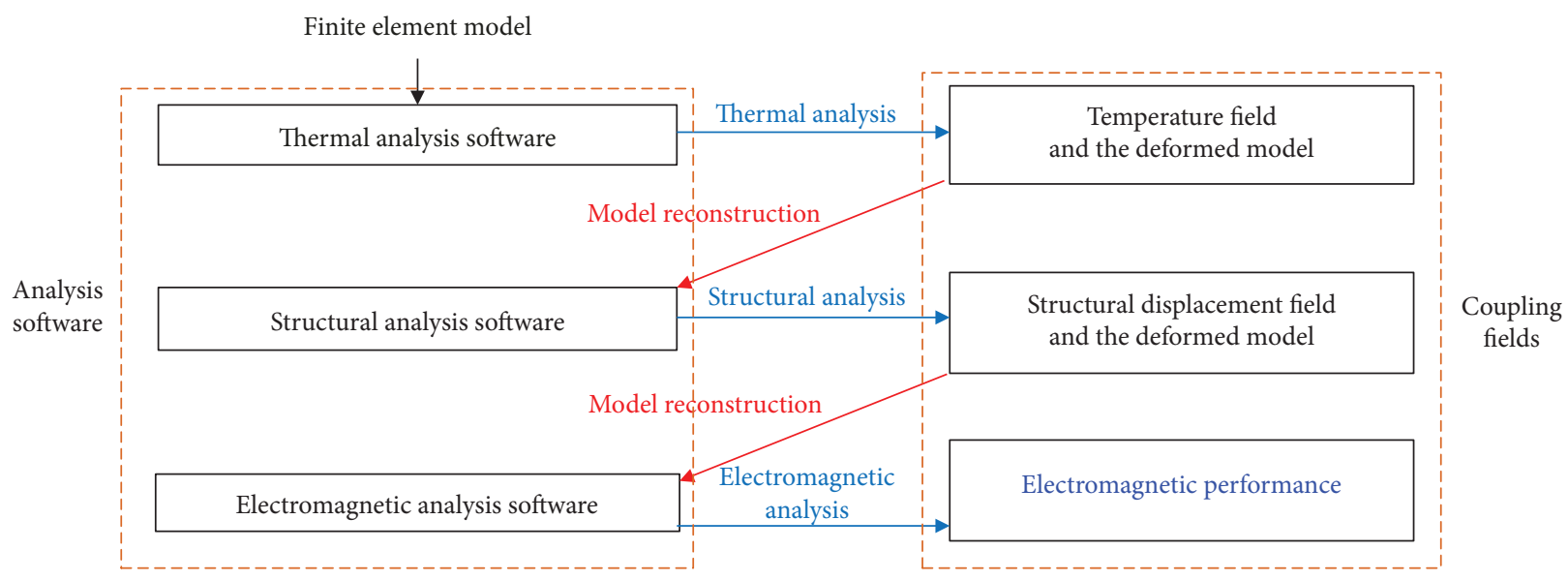

FIgURE 5: The flow chart of the numerical solution.

sequential coupling. Thus, the solution is performed by sequential decoupling [16]. In the traditional method by theoretical model, the actual model was simplified during the numerical solution, which only considers the positional deviations of the array elements and ignores its selfdeformations. This paper performs the numerical solution by using advanced simulation software, which is based on the model reconstruction that takes into account the deformation of the array element. This approach avoids the complicated meshing process and overcomes the problem of large fitting errors in the traditional numerical simulation using simulation software. Figure 5 shows the flow chart of the numerical solution for the multifield coupling problem, and the specific steps are as follows:

(1) The finite element model of the AESA antenna is established. And the thermal load is applied to the model in the thermal analysis software. The temperature field and the deformed model of the antenna are obtained

(2) The deformed model in (1) is introduced into the structural analysis software using the model reconstruction method in this paper (explained in Section 3 ), and it is applied as the external load. The structural displacement analysis is performed. And the structural displacement field and the deformed model superimposed with (1) are obtained

(3) The model superimposed in (2) is introduced into the electromagnetic analysis software still using the model reconstruction method in this paper, and the temperature field in (1) is applied to perform the final electromagnetic performance

\section{Model Reconstruction}

The key to solve the multifield coupling problem of AESA antennas using the simulation software is to overcome the problems that the data models are heterogeneous and the mesh does not match caused by the different analysis tools. In this paper, the model reconstruction of antenna array includes the reconstruction of array elements and the

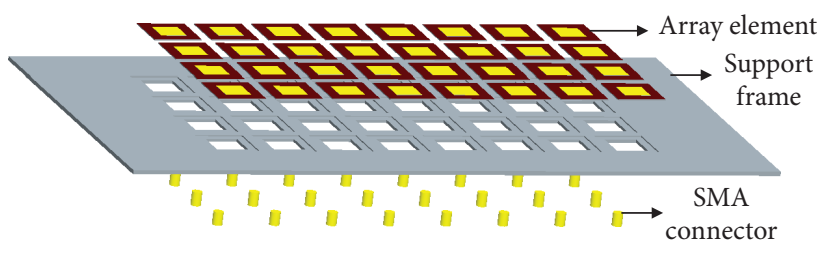

FIGURE 6: Structure of the antenna array.

reconstruction of SMA connectors. Figure 6 shows the structure of the antenna array. And the array elements and feeds are shown in the figure. For the above, the NURBS (nonuniform Rational B-Spline) surface fitting algorithm that guarantees the accuracy of positions and shapes of array elements and the local model reconstruction algorithm that ensures the accuracy of amplitudes and phases of feed connectors are proposed. The flow chart of the reconstruction algorithm is shown in Figure 7.

3.1. Array Element Reconstruction Based on NURBS Surface Fitting. The AESA antenna undergoes various forms of deformation via the different external loads during service. The NURBS surface provides the unified mathematical form for the surfaces representing arbitrary shapes, thereby facilitating the transfer for models through the surface equations and avoiding the geometric modeling errors because of the heterogeneous data models between different coupled fields. At the same time, NURBS technology has been adopted by many excellent CAD/CAM software and has extremely high accuracy for surface fitting [17], thereby improving the fitting accuracy of array element surfaces in the model reconstruction of AESA antennas. The expression of the $p \times q$-ary NURBS surface is [18]

$$
S(u, v)=\frac{\sum_{i=0}^{m} \sum_{j=0}^{n} w_{i, j} d_{i, j} N_{i, p}(u) N_{j, q}(v)}{\sum_{i=0}^{m} \sum_{j=0}^{n} w_{i, j} N_{i, p}(u) N_{j, q}(v)},
$$

where $d_{i, j}, i=0,1, \ldots, m ; j=0,1, \ldots, n$ are the control vertices that correspond to a topological rectangular array that forms a control mesh, $w_{i, j}$ is the weight associated with the vertices, $N_{i, p}(u)$ and $N_{j, q}(v)$ are the canonical B-spline basis 


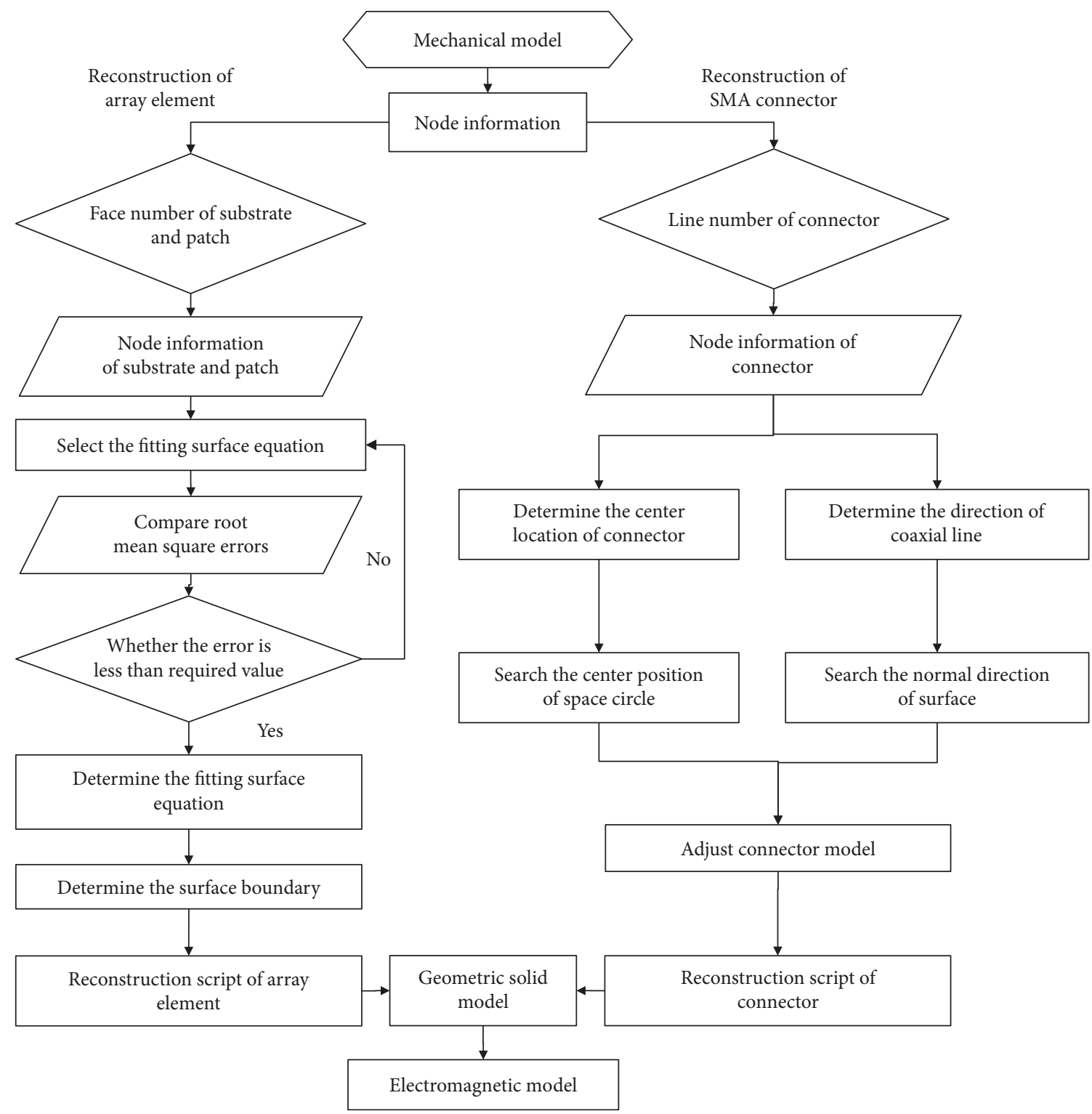

FIGURE 7: Reconstruction algorithm flow chart.

functions determined by the vector $U=\left[u_{0}, u_{1}, \ldots, u_{m-p+1}\right]$ of the direction $u$ and the vector $V=\left[v_{0}, v_{1}, \ldots, v_{n-q+1}\right]$ of the direction $v$, respectively, according to the de Boor-Cox recursion formula. The recursion formula of $N_{i, p}(u)$ is defined as

$$
\left\{\begin{array}{l}
N_{i, 0}(u)=\left\{\begin{array}{l}
1, \quad u_{i} \leq u \leq u_{i+1} \\
0,
\end{array}\right. \\
N_{i, p}(u)=\frac{u-u_{i}}{u_{i+p}-u_{i}} N_{i, p-1}(u)+\frac{u_{i+p+1}-u}{u_{i+p+1}-u_{i+1}} N_{i+1, p-1}(u) .
\end{array}\right.
$$

Postulating $0 / 0=0$, the recursion formula of $N_{j, q}(v)$ is similar.

The specific steps of NURBS surface fitting are as follows:
(1) Assuming that the array of the NURBS surface is $p \times q$-ary and assuming that $w_{i, j}=1$

(2) The node information of the finite element model was extracted as the data points fitted to the NURBS surface. Parameter values were assigned to each data point $p_{i, j}$ that determines the node vector $U$ and $V[19]$

(3) The cross-section curves were constructed from the cross-section data points $p_{i, j}$ on the nodal vector $U$ using the NURBS surface inverse algorithm [18], and the control vertices $\bar{d}_{i, j}(i=0,1, \ldots, m ; j=$ $0,1, \ldots, n-q+1)$ were obtained

(4) The cross-section curves were constructed from the cross-section data points $\bar{d}_{i, j}$ on the nodal vector $V$ using the NURBS surface inverse algorithm, and 


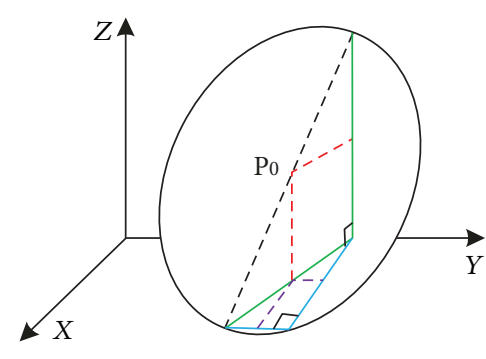

FIGURE 8: Determination of the location of the SMA connector.

the control vertices $d_{i, j}(i=0,1, \ldots, m ; j=0,1, \ldots, n)$ were obtained

(5) The surface was corrected locally and the new weight $w_{i, j}^{*}$ was calculated by the weight changing method [19]

(6) The NURBS surface was obtained based on the calculated control points $d_{i, j}$, the weights $w_{i, j}^{*}$, the node vectors $U$ and $V$, and the surface arrays $p$ and $q$

After obtaining the fitting surface, the surface equation is input into the next physics field in the coupling analysis through the programmed interface program and a new surface is created in the simulation software using the surface equation. In addition, the node information of the model boundary extracted from the previous physical field is used as the constraint condition of the surface equation, which is used to reconstruct the boundary of the array element models. The reconstructed array element model is described in Section 3.3.

3.2. Local Model Reconstruction for the SMA Connector. In order to ensure the accuracy of amplitudes and phases of the reconstructed feed model (SMA connector), this paper discusses the model reconstruction algorithm from two aspects: the location and the pointing direction of SMA connectors. Those two are the important factors affecting the impedance matching between the radiating patch and the feed lines and thus affecting the feeding amplitudes and phases.

Determining the location of the SMA connector, the output port of the connector is approximated as a plane circle. The maximum values and minimum values on the plane circle by the coordinates $x, y$, and $z$ of the nodes are searched, as shown in Figure 8. According to the similar triangle principle, the median value is taken as the point position of the connector. The approximate conditions of the feed port are as follows:

(1) The SMA connector has large rigidity and the actual deformation in service is minimal

(2) Not considering the ductility of the material

Determining the pointing direction of the SMA connector: the surface equation has been obtained in Section 3.1 and written in the general form as $F(x, y, z)=0$. Assuming that the continuity condition is satisfied at $P_{0}\left(x_{0}, y_{0}, z_{0}\right)$ and substituting the coordinates of the position of the SMA connector (also the approximate coordinates of the center of the circle), the normal direction equation of the surface is

$$
\frac{x-x_{0}}{\left.F_{x}\right|_{P_{0}}}=\frac{y-y_{0}}{\left.F_{y}\right|_{P_{0}}}=\frac{z-z_{0}}{\left.F_{z}\right|_{P_{0}}} .
$$

The partial derivatives $F_{x}, F_{y}$, and $F_{z}$ are constructed by substituting the coefficients of the surface equation into the above formula. Moreover, the normal direction at $P_{0}$ $\left(x_{0}, y_{0}, z_{0}\right)$ is obtained as the new pointing direction of the SMA connector. The reconstructed SMA connector model is described in Section 3.3.

3.3. Results and Analysis. In the traditional numerical solutions, some scholars analyzed the multifield coupling of AESA antennas by fitting the solid model in the analysis software and then remeshing on the new model. For example, the Finite Element Modeler (FEM) software is used to complete this work. Figure 9 shows the antenna element model fitted by the FEM software. In the fitting process by the software, the finite element model must first be converted into the form of patch in .x_t format through the surface information of the model. Secondly, the entity model is sewn with the patch, which is a necessary step for FEM to handle the model. In this process, some mesh information and connection information between components are lost and the fitting errors are introduced. Due to the above reasons, the accuracy of the final model is reduced as follows:

(1) As shown in the figure, the cylindrical surface of the SMA connector consists of multiple patches that affect the integrity of the model. This is because of the sparse meshing in the previous structural analysis. However, if the mesh is encrypted, the amount of calculations will be increased. When the model is complex or there are many components, the calculation efficiency will be seriously affected. At the same time, in order to take into account the fitting accuracy and the amount of calculation, the process of mesh adjustment is extremely complicated

(2) As shown in the figure, the upper-end face of the SMA connector is recognized as nonplanar by the electromagnetic analysis software, which makes it impossible to apply the excitation current in the electromagnetic analysis software. This is because of the deformation of the antenna array

(3) As shown in the figure, the lower-end face of the SMA connector and the substrate cannot be completely attached to each other, which affects the feeding effect

In the model reconstruction of this paper, as long as the location and the pointing direction of the SMA connector are accurately determined according to Section 3.2, the standard cylinder in the electromagnetic analysis software can be used to complete the reconstruction of the SMA connector model. Therefore, the model is complete and regular and its lower-end surface is plane. In addition, the substrate and 


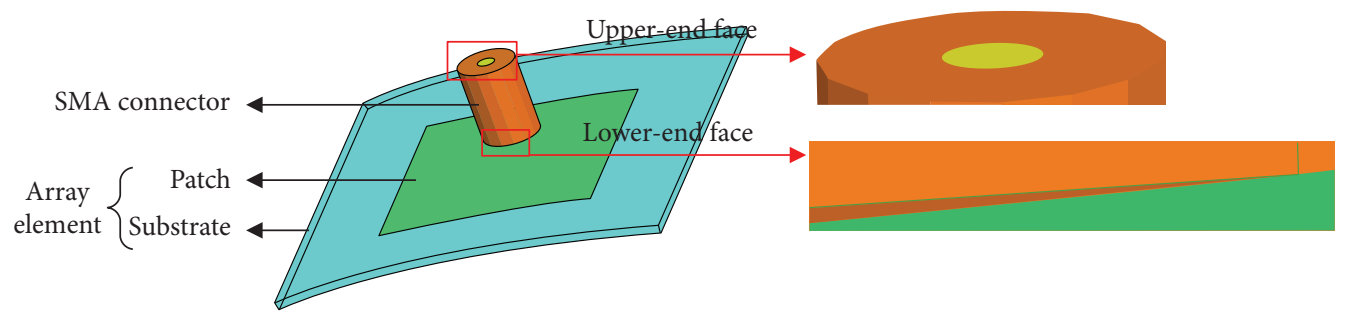

Figure 9: Antenna element model fitted by the FEM software.

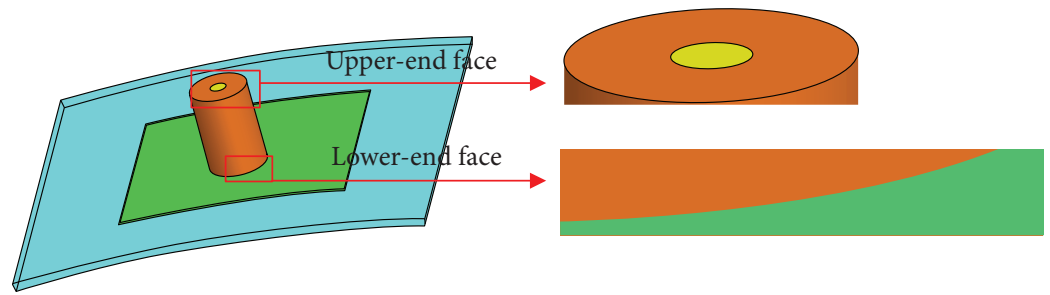

(a) Element model generated by the model reconstruction method

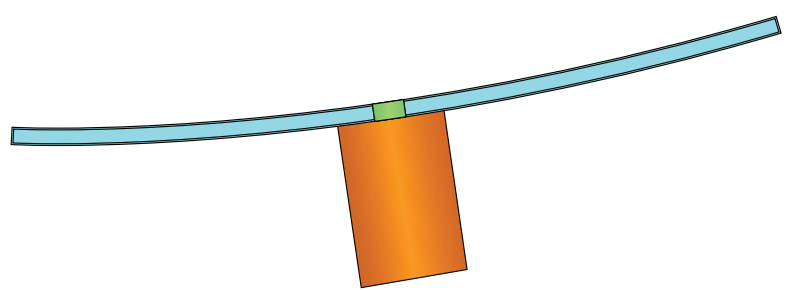

(b) Side view

Figure 10: Antenna element model generated by the model reconstruction method.

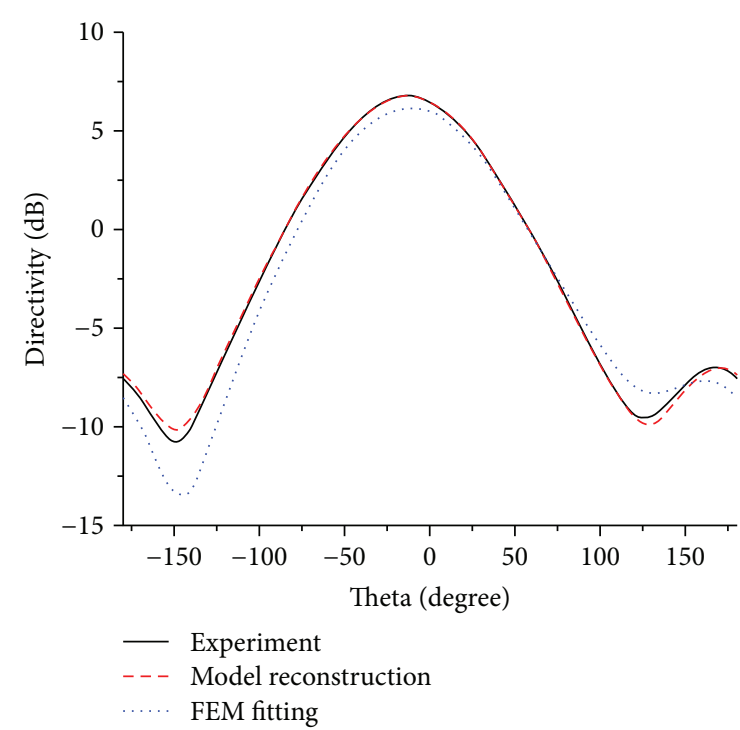

(a) Radiation pattern

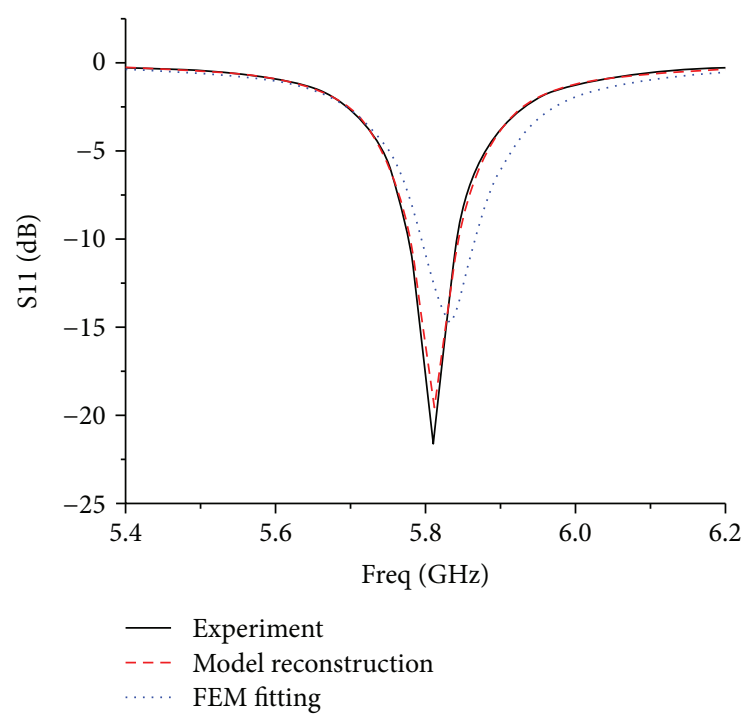

(b) Return loss

FIGURE 11: Electrical performance results.

the SMA connector need to be subtracted by the Boolean subtraction operation in the analysis software. After the subtraction, the curvature of each point on the upper-end surface of the SMA connector coincides with the curvature of each point on the substrate and a perfect fit between the two is guaranteed. At the same time, the NURBS surface fitting algorithm ensures the accuracy of the array element model. Figure 10(a) shows the antenna element model generated by the model reconstruction method in this paper, and Figure 10(b) shows the side view.

The results of the electrical performance obtained by the FEM fitting method and the model reconstruction method were compared with the experimental test results, respectively. Figure 11(a) shows the radiation pattern and Figure 11(b) 

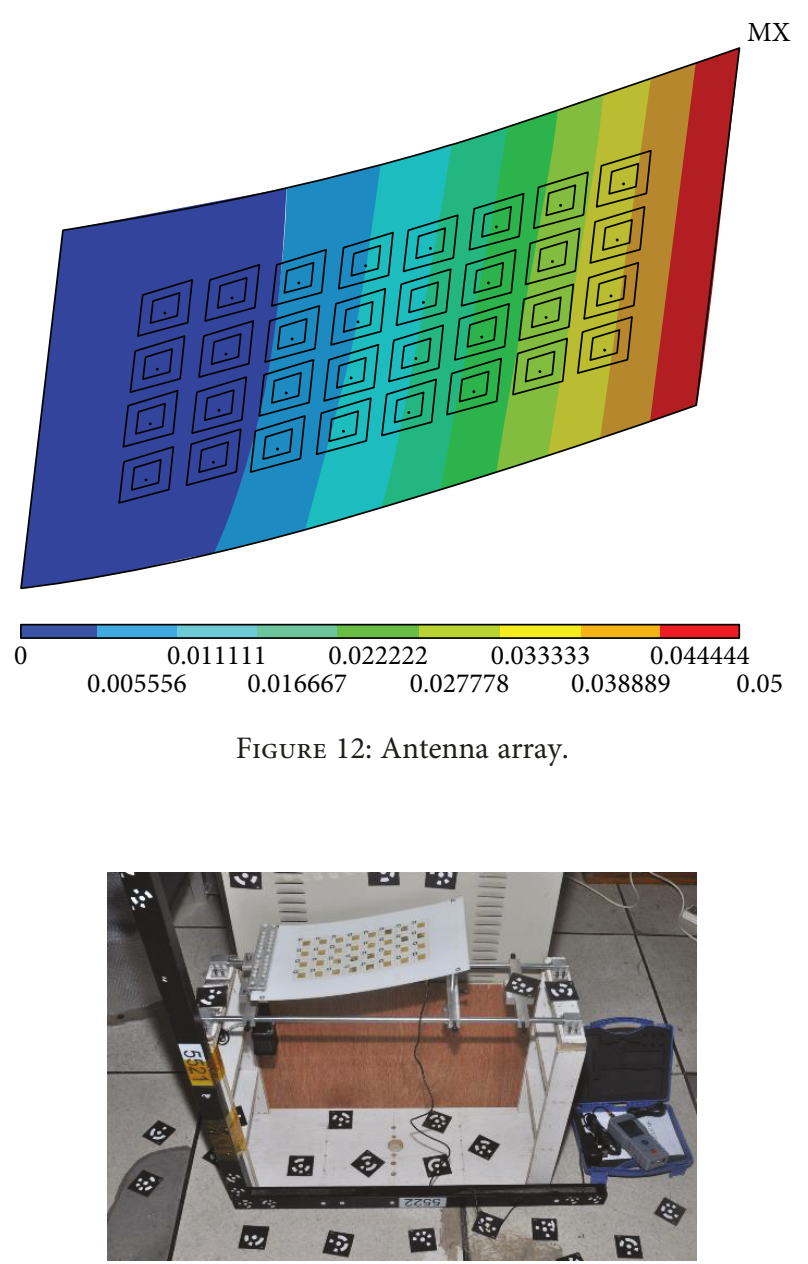

FIgURE 13: Testing experiment of material parameters.

shows the return loss. The results show that the model reconstruction method has higher analysis accuracy.

\section{Simulations and Experiment Results}

The following is the case of multifield coupling analysis of the 32-element AESA antenna whose operating frequency is $5.8 \mathrm{GHz}$ in C-band. The measurements and the comparison of simulations by different methods were shown.

\subsection{Simulation Process Based on Model Reconstruction}

4.1.1. Analysis of the Structural Displacement Field and Temperature Field. As described in Section 2.2, the analysis of the structural displacement field and the temperature field was performed in ANSYS. In this case, the left-end face of the antenna was fixed and the displacement of $50 \mathrm{~mm}$ (close to a wavelength of the $5.8 \mathrm{GHz}$ antenna) was applied to the rightend face. At the same time, the temperature load was applied in the form of body load. Superimposing the force load and temperature load, the structure displacement field distribution and temperature field distribution of the antenna were obtained. And the deformation of the antenna array was extracted for the subsequent model reconstruction and electrical performance analysis, as shown in Figure 12 . Figure 13 shows the material parameter testing experiment. The element types and material properties are shown in Table 1.

4.1.2. Model Reconstruction and Electrical Performance Analysis. According to the method described in Section 3, the model was reconstructed in the electromagnetic analysis software by the node information of the finite element model in the temperature and structural displacement fields. Meshing and electrical performance analysis were performed on the reconstruction model. Figure 14 shows the antenna entity model reconstructed in the electromagnetic field. The model is complete and accurate and can be directly used for simulation analysis. Figure 14(a) shows the front view, where the reconstructed model of the substrate and the patch is regular and accurate and the two are completely attached to each other. Figure 14(b) shows the rear view, where the position of the SMA connector is accurate and the direction of the coaxial line is the same as the normal of the surface. The analysis results of electrical performance are described in Section 4.2.

4.2. Comparisons of Simulations and Experiment Results. The electrical performance of the antenna array was measured, as shown in Figure 15. Figure 16 shows the antenna array model [20].

4.2.1. Analysis of Electrical Performance without Considering Field Coupling. Without considering the field coupling, the radiation pattern obtained by the method in this paper was compared with experimental test results, as shown in Figure 17. By comparison, the relative error of the gain between the two is $1.92 \%$, the relative error of the beam pointing is $0 \%$, and the relative error of the sidelobe level is less than 9.90\%, as shown in Table 2. The experimental results illustrate the accuracy of this method.

4.2.2. Analysis of Electrical Performance in the Coupling Field. In the coupling field, the results of the model reconstruction method and other traditional methods were compared with the measured results as shown in Figure 18. These methods were compared from the five aspects: analysis accuracy, calculation time, applicability for AESA antenna, automation capability, and coupling analysis capability. First, Table 3 shows that the model reconstruction method has the highest accuracy compared with the other traditional methods. As follows, the relative error of gain is $2.24 \%$, the relative error of beam pointing (ratio relative to the beam width) is $2.17 \%$, and the relative error of the sidelobe is less than $8.71 \%$. Secondly, Table 4 shows the comparisons from other aspects. It shows that the model reconstruction method is superior to other methods: (1) The model reconstruction method avoids the tedious meshing process and saves much time in computing. (2) The model reconstruction method has good applicability for the structure of AESA antenna, which is capable to describe both connection of components and self-deformation of elements accurately. (3) The model reconstruction method has great automation capability, thereby improving the efficiency of the solution. (4) The 
TABLE 1: Element types and material attribute parameters.

\begin{tabular}{lccccc}
\hline Component & Material attribute & Element type & Elastic modulus $(\mathrm{Mpa})$ & Poisson's ratio & Density $\left(\mathrm{kg} / \mathrm{m}^{3}\right)$ \\
\hline Framework & Rogers5880 & SOLID185 & 33,500 & 0.38 & 1130 \\
Substrate & Rogers4350 & SOLID185 & 1200 & 0.36 & 565 \\
Patch & Copper & SOLID185 & $1.08 e 11$ & 0.33 & 8900 \\
Feed core & Copper & SOLID185 & $1.08 e 11$ & 0.33 & 8900 \\
Feed housing & Rogers5880 & SOLID185 & 33,500 & 0.38 & 1130 \\
\hline
\end{tabular}

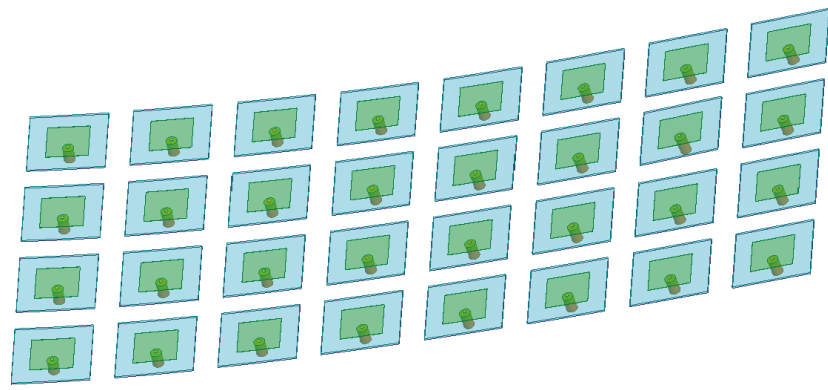

(a) Front view of the reconstructed antenna array

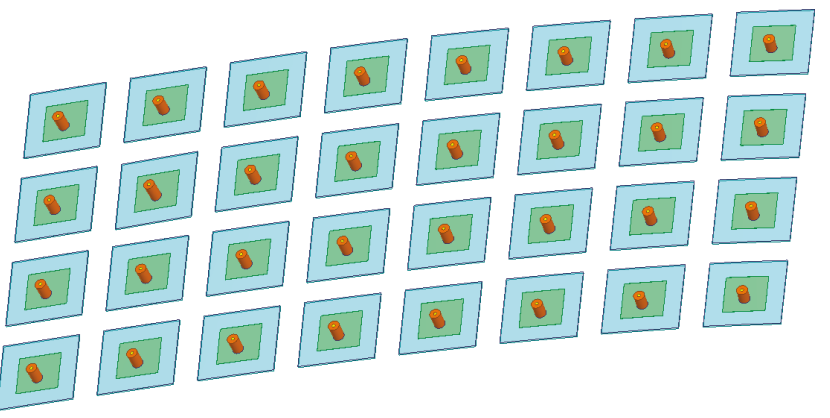

(b) Rear view of the reconstructed antenna array

FIgURE 14: Reconstructed antenna array model.

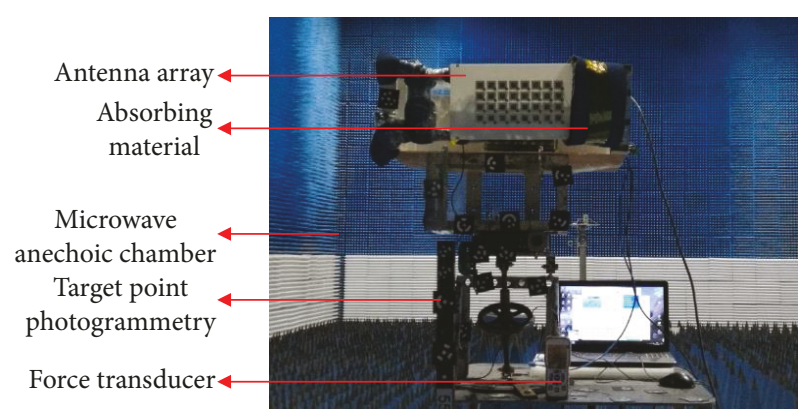

FIgURE 15: Electrical performance tester.

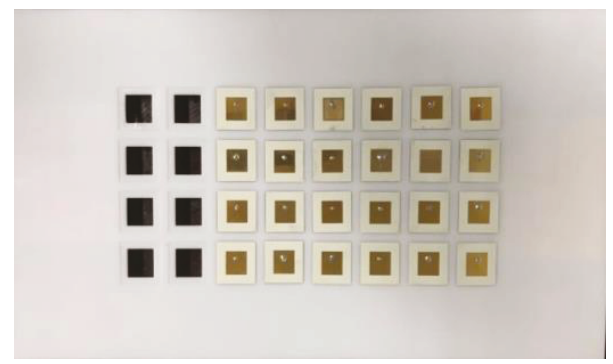

FIgURE 16: Antenna array model.

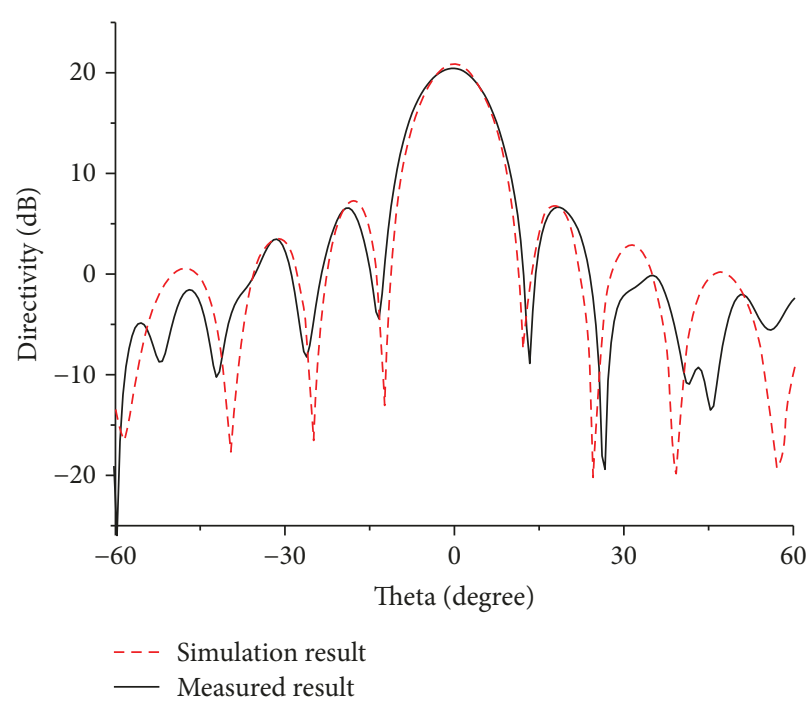

FIGURE 17: Radiation pattern without considering field coupling.

TABLE 2: Comparison of measured and simulation results.

\begin{tabular}{lcccc}
\hline & Gain $(\mathrm{dB})$ & $\begin{array}{c}\text { Beam } \\
\text { direction }\left({ }^{\circ}\right)\end{array}$ & \multicolumn{2}{c}{$\begin{array}{c}\text { Sidelobe } \\
(\mathrm{dB})\end{array}$} \\
\hline Simulation & 20.44 & 0 & 6.56 & 6.63 \\
Experiment & 20.84 & 0 & 7.28 & 6.91 \\
Relative error (\%) & 1.92 & 0 & 9.90 & 4.05 \\
\hline
\end{tabular}

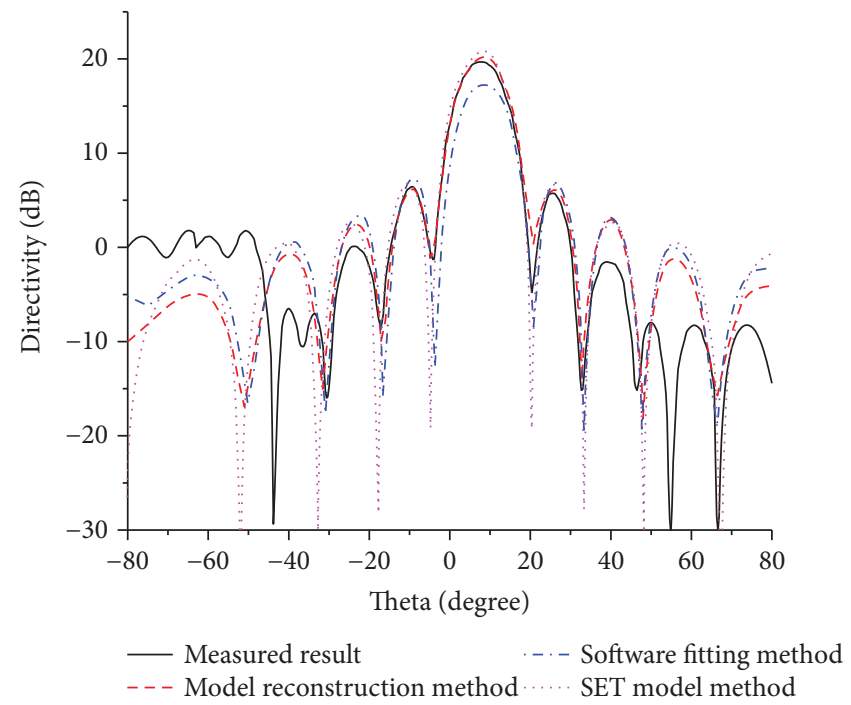

FIGURE 18: Radiation pattern in the coupling field. 
TABLE 3: Comparison between solution results using different methods.

\begin{tabular}{|c|c|c|c|c|}
\hline & Gain $(\mathrm{dB})$ & Beam direction $\left({ }^{\circ}\right)$ & Left sidelobe $(\mathrm{dB})$ & Right sidelobe $(\mathrm{dB})$ \\
\hline Measured result & 19.68 & 7.46 & 6.43 & 5.74 \\
\hline Model reconstruction & 20.12 & 7.73 & 6.29 & 6.24 \\
\hline Relative error compared with experiment (\%) & 2.24 & 2.17 & 1.97 & 8.71 \\
\hline Software fitting & 17.45 & 8.70 & 7.26 & 6.89 \\
\hline Relative error compared with experiment (\%) & 11.33 & 11.48 & 12.91 & 20.03 \\
\hline SET model & 20.80 & 7.80 & 6.75 & 6.75 \\
\hline Relative error compared with experiment (\%) & 5.69 & 3.06 & 4.98 & 17.60 \\
\hline
\end{tabular}

TABLE 4: Comparison of different methods in multifield coupling analysis.

\begin{tabular}{lccc}
\hline & Model reconstruction & Software fitting & SET model \\
\hline Calculation time $(\mathrm{s})$ & 3020 & 43200 & 3650 \\
$\begin{array}{l}\text { Applicability for } \\
\text { AESA antenna }\end{array}$ & $\begin{array}{c}\text { Describing both connection of } \\
\text { components and self-deformation } \\
\text { of elements }\end{array}$ & $\begin{array}{c}\text { Unable to describe connection } \\
\text { of components }\end{array}$ & $\begin{array}{c}\text { Unable to describe self-deformation } \\
\text { of elements }\end{array}$ \\
Automation capability & Fully automated & Unable to be automated & $\begin{array}{c}\text { Unable to be automated in the } \\
\text { process of model simplification } \\
\text { and equivalence }\end{array}$ \\
Coupling analysis capability & $\begin{array}{c}\text { Both multifield coupling } \\
\text { and field-circuit coupling }\end{array}$ & $\begin{array}{c}\text { Difficult to analyze multifield } \\
\text { coupling and unable on analyzing } \\
\text { field-circuit coupling }\end{array}$ & $\begin{array}{c}\text { Unable to analyze field-circuit } \\
\text { coupling }\end{array}$ \\
\hline
\end{tabular}

model reconstruction method could to be used to analyze both multifield coupling and field-circuit coupling.

\section{Conclusion}

In this paper, the field-circuit coupling model was established by introducing the deformation errors of the feed system into the existing theoretical model. In addition, the new numerical solution method for the multi-field-coupled problem of AESA antennas based on model reconstruction was proposed. In the model reconstruction, two algorithms were presented: the NURBS (nonuniform rational B-spline) surface fitting algorithm that completes the mapping from finite element models to geometric models by the surface equations established by the node information and the local model reconstruction algorithm that determines the local geometric models by the positions and the directions. The numerical solution was applied to the 32-element AESA antenna. And the comparisons between the simulations and measured results shows the following: (1) Compared with the traditional fitting simulation software, the proposed method avoids the fussy meshing process and saves much time in computing. (2) Compared with the traditional coupled SET model, the proposed method has high accuracy. This is because the NURBS surface fitting algorithm guarantees the accuracy of both the positions and shapes of array elements and the local model reconstruction algorithm ensures the accuracy of the amplitudes and phases of feed connectors. Furthermore, the method proposed in this paper provides a new idea for the numerical solution of the multi-fieldcoupled problem of AESA antennas.

\section{Data Availability}

The data used to support the findings of this study are available from the corresponding author upon request.

\section{Conflicts of Interest}

The authors declare that they have no conflicts of interest.

\section{Acknowledgments}

This work was supported by the National Natural Science Foundation of China (nos. 51575419, 51775405, and 51490664), the Joint Funds of Ministry of Education of the People's Republic of China (no. 6141A02022107), and Defense Basic Research Program (no. JCKY2016210B002). Zhiheng Cai, Bo Tang, Le Kang, Zhanbiao Yang, and Siwen Zhang from Xidian University provided experimental help during the project. Their efforts were crucial to the completion of the research.

\section{References}

[1] T. Lambard, O. Lafond, M. Himdi, H. Jeuland, S. Bolioli, and L. le Coq, "Ka-band phased array antenna for high-data-rate SATCOM," IEEE Antennas and Wireless Propagation Letters, vol. 11, no. 1, pp. 256-259, 2012.

[2] C. Luison, A. Landini, P. Angeletti et al., "Aperiodic arrays for Spaceborne SAR Applications," IEEE Transactions on Antennas and Propagation, vol. 60, no. 5, pp. 2285-2294, 2012.

[3] M. Y. Chen, D. Pham, H. Subbaraman, X. lu, and R. T. Chen, "Conformal ink-jet printedC-band phased-array antenna incorporating carbon nanotube field-effect transistor based 
reconfigurable true-time delay lines," IEEE Transactions on Microwave Theory and Techniques, vol. 60, no. 1, pp. 179184, 2012.

[4] B. Y. Duan, "Review of electromechanical coupling of electronic equipment," Scientia Sinica Informationis, vol. 45, no. 3, pp. 299-312, 2015.

[5] T. Takahashi, N. Nakamoto, M. Ohtsuka et al., "On-board calibration methods for mechanical distortions of satellite phased array antennas," IEEE Transactions on Antennas and Propagation, vol. 60, no. 3, pp. 1362-1372, 2012.

[6] H. Kamoda, J. Tsumochi, T. Kuki, and F. Suginoshita, "A study on antenna gain degradation due to digital phase shifter in phased array antennas," Microwave and Optical Technology Letters, vol. 53, no. 8, pp. 1743-1746, 2011.

[7] B. Y. Duan, H. Qiao, and L. Z. Zeng, "The multi-field-coupled model and optimization of absorbing material's position and size of electronic equipments," Journal of Mechatronics and Applications, vol. 2010, Article ID 569529, 6 pages, 2010.

[8] C. S. Wang, W. F. Wang, B. Y. Duan et al., "Integrated radiation-scattering optimization of active phased array antennas based structural-electromagnetic coupling method," Acta Electronica Sinica, vol. 43, no. 6, pp. 1185-1191, 2015.

[9] C. S. Wang, B. Y. Duan, F. S. Zhang, and M. B. Zhu, "Coupled structural-electromagnetic-thermal modelling and analysis of active phased array antennas," IET Microwaves, Antennas \& Propagation, vol. 4, no. 2, pp. 247-257, 2010.

[10] Y. Li, S. X. Zhang, F. S. Zhang, J. P. Shang, and L. W. Zou, "Accurate measurement of XPD for the microwave antenna using the near field method," Journal of Xidian University, vol. 27, no. 2, pp. 224-227, 2000.

[11] M. Chen, F. Zheng, and N. Li, "Mesh mapping of large deployable reflector in mechanical-electromagnetic analyses," Advanced Materials Research, vol. 460, pp. 43-47, 2012.

[12] P. Li, F. Zheng, and X. Ji, "Electromechanical coupled analysis of large broad band reflector antennas," Journal of Xidian University, vol. 36, no. 3, pp. 473-479, 2009.

[13] M. G. Wang, S. W. Lv, and R. X. Liu, Antenna Array Analysis and Synthesis, University of Electronic Science and Technology, 1989.

[14] J. C. Bolomey and F. E. Gardiol, Engineering Applications of the Modulated Scatter Technique, Microwaves \& RF, 2001.

[15] T. H. Liu and K. F. Tsang, "The developments of dualfrequency/dual-polarized patch antennas," Modern Radar, vol. 21, no. 5, pp. 91-99, 1999.

[16] B. Y. Duan and M. Wang, "Research of the theoretical model of multi-field coupling and multidisciplinary optimization design on microwave antennas," Acta Electronica Sinica, vol. 41, no. 10, pp. 2051-2060, 2013.

[17] F. Z. Shi, Computer Aided Geometric Design and Non-Uniform Rational B-Spline, Higher Education Press, 2001.

[18] L. Piegl, "On NURBS: a survey," IEEE Computer Graphics and Applications, vol. 11, no. 1, pp. 55-71, 1991.

[19] R. Ding, "Algorithm of NURBS surface fitting and its application," Journal of Tianjin University of Technology and Education, vol. 14, no. 4, pp. 30-32, 2004.

[20] J. Zhou, H. Li, L. Kang, B. Tang, J. Huang, and Z. Cai, “Design, fabrication, and testing of active skin antenna with 3D printing array framework," International Journal of Antennas and Propagation, vol. 2017, Article ID 7516323, 15 pages, 2017. 


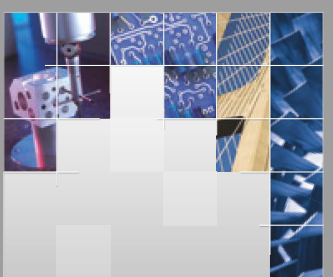

\section{Enfincering}
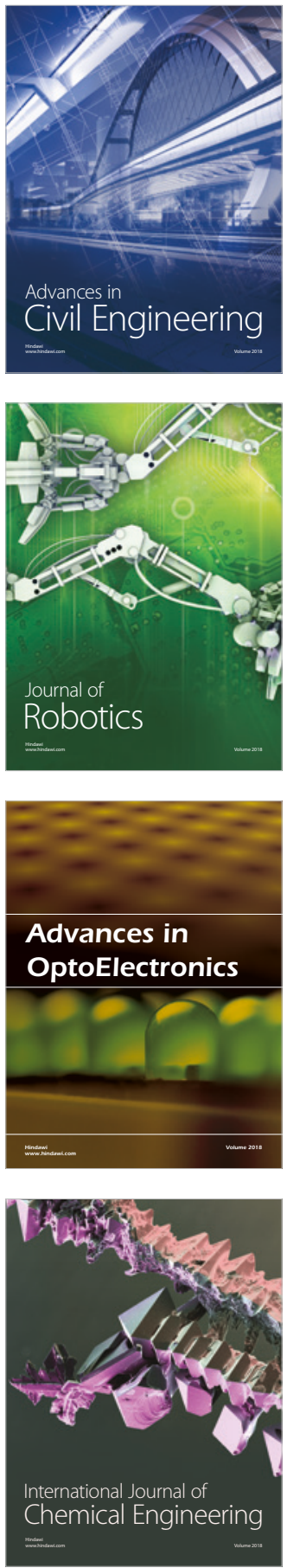

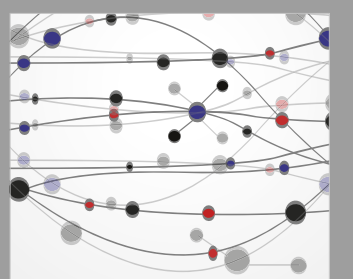

\section{Rotating \\ Machinery}

The Scientific World Journal

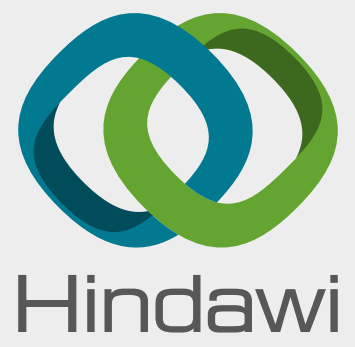

Submit your manuscripts at

www.hindawi.com
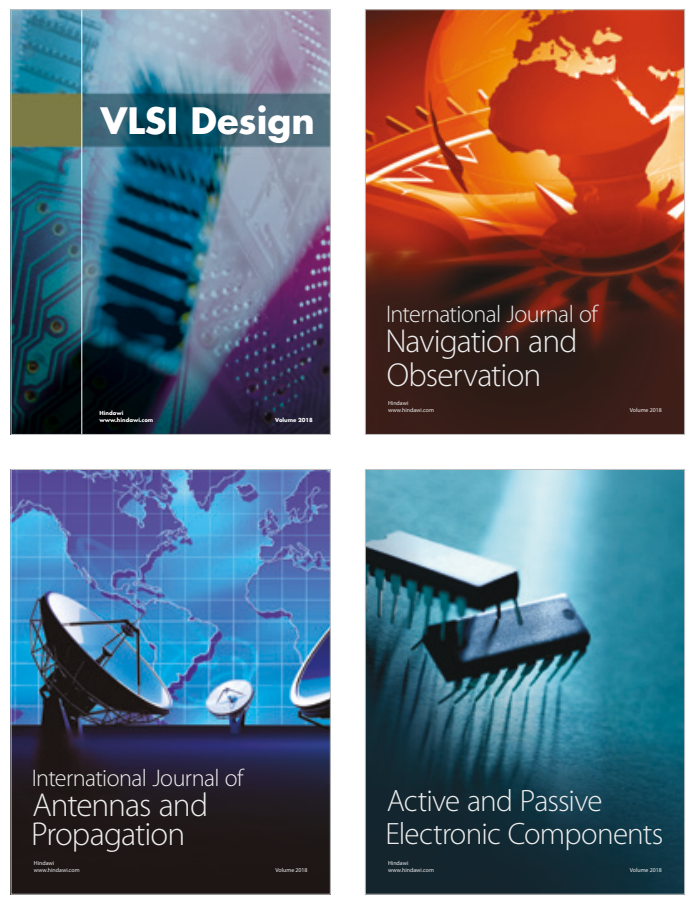
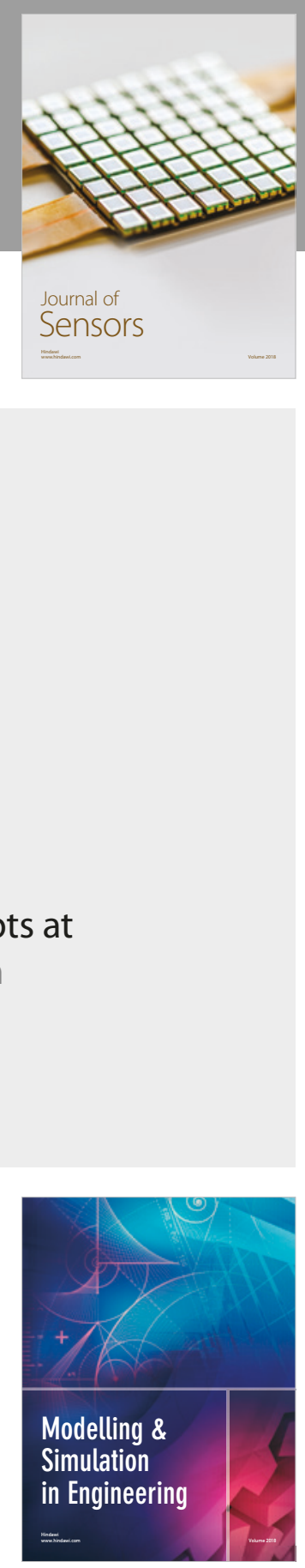

\section{Advances \\ Multimedia}
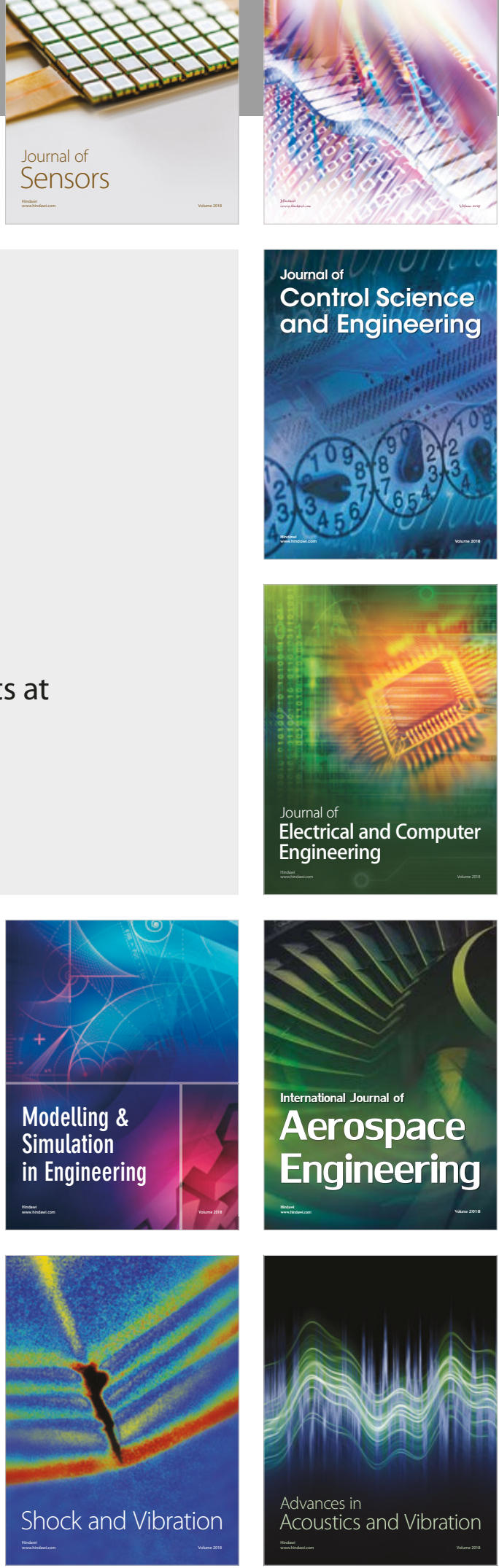